\title{
OCLUSÃO DE FÍSTULA ORONASAL EM GATO COM USO SOMENTE DE RESINA ACRÍLICA AUTOPOLIMERIZÁVEL
}

\author{
Reginaldo Pereira de Sousa Filho ${ }^{1 *}$ \\ Keytyanne de Oliveira Sampaio ${ }^{1}$ \\ Janaina Serra Azul Monteiro Evangelista ${ }^{1}$ \\ Marina Gabriela Monteiro Carvalho Mori da Cunha ${ }^{1}$
}

SOUSA FILHO, R. P. de; SAMPAIO, K. de O.; EVANGELISTA, J. S. A. M.; CUNHA, M. G. M. C. M. da. Oclusão de fístula oronasal em gato com uso somente de resina acrílica autopolimerizável. Arq. Ciênc. Vet. Zool. UNIPAR, Umuarama, v. 19, n. 2, p. 101-105, abr./jun. 2016.

RESUMO: A fenda palatina é uma comunicação congênita ou adquirida entre as cavidades oral e nasal, permitindo a passagem de alimentos e líquidos entre elas. O diagnóstico é feito por meio do exame físico da cavidade oral, sendo o tratamento baseado em técnicas cirúrgicas. Relata-se um caso bem-sucedido de oclusão de fístula oronasal utilizando somente resina acrílica autopolimerizável em um felino com histórico de espirros frequentes, secreção nasal purulenta crônica, halitose severa, emagrecimento e trauma sofrido há dois anos, que culminara em fratura da sínfise mandibular corrigida no mesmo período por cerclagem com fios de aço. No exame clínico verificou-se secreção nasal muco-purulenta fétida e a presença de uma grande fístula palatina na extensão do palato duro. Encaminhou-se o paciente para realização dos exames pré-cirúrgicos para posterior correção da fistula oronasal com o uso de resina acrílica autopolimerizavel. O procedimento foi bem sucedido visto que o animal deixou de apresentar os sinais clínicos e voltou a alimentar-se normalmente. A técnica mostrou-se eficaz e de fácil execução para correção de fenda palatina traumática de grande extensão em gato, na qual não se utilizou suturas ou flaps, pois os mesmos não eram praticáveis, podendo ser utilizada em substituição a outros métodos convencionais.

PALAVRAS-CHAVE: Felino. Fenda palatina. Trauma.

\section{ORONASAL FISTULA OCCLUSION IN CAT USING ONLY ACRYLIC RESIN}

\begin{abstract}
Cleft palate is a congenital or acquired communication between the oral and nasal cavities which can often contaminate the nasal cavity with saliva or food. The diagnosis is made by physical examination of the oral cavity and it is usually treated with surgery. We report a successful case of oronasal fistula occlusion using only acrylic resin in a feline. The patient presented with a history of frequent sneezing, chronic purulent nasal discharge, severe halitosis, weight loss and facial trauma suffered two years ago. The animal had mandibular symphysis fracture that was corrected by cerclage with steel wires. On clinical examinationit was observedthe presence of smelling nasal mucus, purulent secretions and the presence of a large cleft fistula in the extension of the hard palate. The patient was submitted to surgery in order to repair the oronasal fistula using acrylic resin. The surgery was curative eliminating clinical signs. The technique was effective and easy to be performed in this case of a traumatic cleft palate with large deffect in a cat. This technique can be used to replace other conventional methods, mainly in cases that the use of sutures or flaps are not feasible.
\end{abstract}

KEYWORDS: Cleft palate. Feline. Trauma

\section{OCLUSIÓN DE FÍSTULA ORONASAL EN GATO CON USO SÓLO DE RESINA ACRÍLICA AUTOPOLIMERIZÁVEL}

RESUMEN: El paladar hendido es una comunicación congénita o adquirida entre las cavidades oral y nasal, lo que permite el paso de alimentos y líquidos entre ellos. El diagnóstico se realiza mediante un examen físico de la cavidad oral, siendo el tratamiento basado en técnicas quirúrgicas. Presentamos un caso de éxito de oclusión de la fístula oronasal usando sólo la resina acrílica autopolimerizável en un felino con histórico de frecuentes estornudos, secreción nasal purulenta crónica, halitosis severa, pérdida de peso y trauma sufrido hace dos años, culminando en fractura de la sínfisis mandibular, corregida en el mismo período por cerclaje con hilos de acero. En el examen clínico se verificó moco nasal maloliente, secreciones purulentas y la presencia de una gran fístula palatina en la extensión del paladar duro. Se hicieron exámenes pre-quirúrgicos para la posterior corrección de la fístula oronasal, mediante resina acrílica autopolimerizavel. El procedimiento fue exitoso porque el animal dejó de presentar signos clínicos y volvió a alimentarse normalmente. La técnica fue eficaz y de fácil ejecución para corrección de paladar hendido traumático de gran longitud en gato, en la cual no se utilizó suturas o aletas, ya que no eran viables y puede ser utilizado para sustituir a otros métodos convencionales.

PALABRAS CLAVE: Felino. Paladar hendido. Trauma.

DOI: https://doi.org/10.25110/arqvet.v19i2.2016.5929

${ }^{1}$ Universidade de Franca. Endereço para correspondência: Pós-Graduação em Ciência Animal, Hospital Veterinário, Universidade de Franca (UNIFRAN). Av. Armando Salles de Oliveira, 201, Jd. Universitário, Franca- SP, CEP 14404-600. E-mail: dennerbiovet@hotmail.com

${ }^{2}$ Universidade Federal do Mato Grosso do Sul. 


\section{Introdução}

A fenda palatina é uma comunicação oronasal patológica que permite a passagem de alimentos e líquidos da cavidade oral para a nasal (GRIFFITHS; SULLIVAN, 2001). Pode ser observada isolada nopalato primário (lábio e pré maxila) ou associada às anomalias do palato secundário (palatos duro e mole) (GIOSO, 2003).

As fendas palatinas podem ser de origem congênita ou adquirida, sendo que as adquiridas podem ser causadas por trauma ou doença (SIVACOLUNDHU, 2007). As de origem congênita pode esta relacionada com fatores hereditários, ingestão de medicamentos, agentes químicos, ou plantas tóxicas teratogênicas durante a gestação, e interferência mecânica com o embrião em desenvolvimento (VERSTRAETE; LOMMER, 2012).

Os sinais clínicos apresentados estão associados com a idade do animal e variam entre dificuldade para deglutir, tosse, espirros, drenagem de alimento por via nasal,corrimento nasal e infecções frequentes no trato respiratório. Animais recém-nascidos devem ser alimentados por sonda, visto que facilita o reparo da fistula por permitir a proliferação tecidual, além de permitir a nutrição do paciente até que possam ser submetidos ao procedimento cirúrgico (GIOSO, 2003).O diagnóstico da afecção é realizado mediante o exame minucioso da cavidade oral (FOSSUM, 2002).

A técnica cirúrgica escolhida dependerá da dimensão da lesão e da facilidade de acesso à região afetada (MANFRA, 2012). A maioria das cirurgias realizadas são baseadas em técnicas de Flap (BELOTI et al., 2011), como a técnica de retalho de avanço palato mole (ROCHA;BECKMAN, 2010), a transferência microvascular de cartilagem (CONTESINI et al., 2003), a utilização de tela de polipropileno (SILVA et al., 2009), a utilização de próteses de resina acrílica autopolimerizável (SMITH, 2000; ROEHSIG et al., 2001; LEE et al., 2006) e de placa de titânio pré-modelada (KUIPERS et al., 2012). Sivacolundhu, (2007) e Souza et al. (2007) faz referência à utilização de enxertos mucoperiosteais, de enxertos da mucosa palatal ou gengival ou lingual, a aplicação de próteses de resina acrílica autopolimerizável.

O sucesso da cirurgia depende da preservação da vascularização dos enxertos e da capacidade do enxerto resistir ao stress mecânico induzido pela mastigação, deglutição e movimentação traumática permanente da língua no palato regional (SIVACOLUNDHU, 2007).

O presente trabalho tem como objetivo relatar um caso bem-sucedido de oclusão de fístula oronasal, utilizando somente resina acrílica autopolimerizável.

\section{Relato do Caso}

Um felino macho, castrado, sem raça definida, com três anos de idade, $3 \mathrm{KG}$, foi atendido em uma clínica veterinária particular na cidade de Fortaleza- CE, apresentando como queixa principal a presença de espirros frequentes, secreção nasal purulenta crônicos, halitose severa e emagrecimento progressivo. O proprietário relatou ainda que o felino havia sofrido um trauma dois anos antes, quando foi submetido à redução de fratura de sínfise mandibular por cerclagem com fios de aço. $\mathrm{O}$ animal alimentava-se somente de ração seca comercial
No exame clínico geral percebeu-se que o animal apresentava pelo opaco e secreção nasal muco-purulenta fétida. Na inspeção da cavidade oral foi observada a presença de uma grande fístula palatina, com dimensões $3 \times 4,5 \mathrm{~cm}$, atingindo quase $2 / 3$ da extensão do palato duro (Figura 1). Na cavidade nasal foi encontrado resto alimentares em decomposição. Não foi encontrado alterações na ausculta torácica. Foi solicitado exames pré-cirúrgicos, tais como hemograma, fostatase alcalina, creatinina e albumina séricas, radiografia torácica e eletrocardiograma. Observou-se alteração apenas no hemograma, o qual evidenciou leucocitose com desvio à direita e monocitose.

Utilizou-se cloridrato de tramadol, na dose de $1 \mathrm{mg} /$ $\mathrm{kg}$ IM e o midazolan, $0,5 \mathrm{mg} / \mathrm{kg}$ IM como medicações pré anestésicas, como antibiótico profilaxia utilizou-se ceftriaxona sódica, $25 \mathrm{mg} / \mathrm{kg} \mathrm{IV}, 15$ minutos antes da indução da anestesia, que foi feita com propofol, na dose de $4 \mathrm{mg} / \mathrm{kg}$, IV. $\mathrm{O}$ animal foi entubado e mantido em plano anestésico com isoflorano em oxigênio $100 \%$, Com o cuff da sonda devidamente inflado, realizou-se uma criteriosa anti-sepsia com solução aquosa de clorexidine a $0,12 \%$ e debridamento da área da fístula, inclusive de toda cavidade nasal, retirando-se grande quantidade de tecido necrótico e restos alimentares. Introduziu-se uma sonda uretral humana número 14 em cada narina, até serem visualizadas na faringe a fim de impedir a obliteração total da cavidade nasal com a aplicação da resina.

Misturou-se o copolímero em pó com o monômero líquido na quantidade necessária para preencher o espaço da fístula, até que a resina atingisse uma maleabilidade ideal para o implante no local. Preencheu-se o defeito com o material polimerizável, tendo-se o cuidado de irrigar toda a região com solução de cloreto de sódio $0,9 \%$ resfriada, a fim de diminuir a temperatura nos tecidos em contato com a resina. Após a aplicação, ajuste, amoldamento e retirada do excesso do material na cavidade oral (Figura 2), movimentou-se gentilmente as sondas uretrais, enquanto a resina polimerizava-se.

No período pós- operatório foi prescrito antibioticoterapia com azitromicina, $5 \mathrm{mg} / \mathrm{kg}$, SID, durante sete dias e meloxican, $0,05 \mathrm{mg} / \mathrm{kg}$, SID, por três dias. Recomendou-se o uso de colar-elisabetano e o fornecimento de dieta úmida, com ração comercial, durante 10 dias.

O animal retornou com 10 dias após o procedimento, já apresentando diminuição dos episódios de espirros, mas ainda com secreção nasal serosa. $\mathrm{O}$ apetite havia melhorado e houve um aumento de $500 \mathrm{~g}$ no peso total. Recomendou-se portanto, o retorno à alimentação com ração sólida. Após seis meses da implantação da resina, o gato retornou, quando verificou-se a total integridade do implante, sem sinais clínicos de mobilidade do mesmo ou inflamação nos tecidos adjacentes. Clinicamente o gato estava bem, pesando $4,5 \mathrm{~kg}$, sem sinais de enfermidade respiratória (Figura 3). 
Figura 1: Felino macho com fístula palatina traumática em quase toda extensão do palato duro.

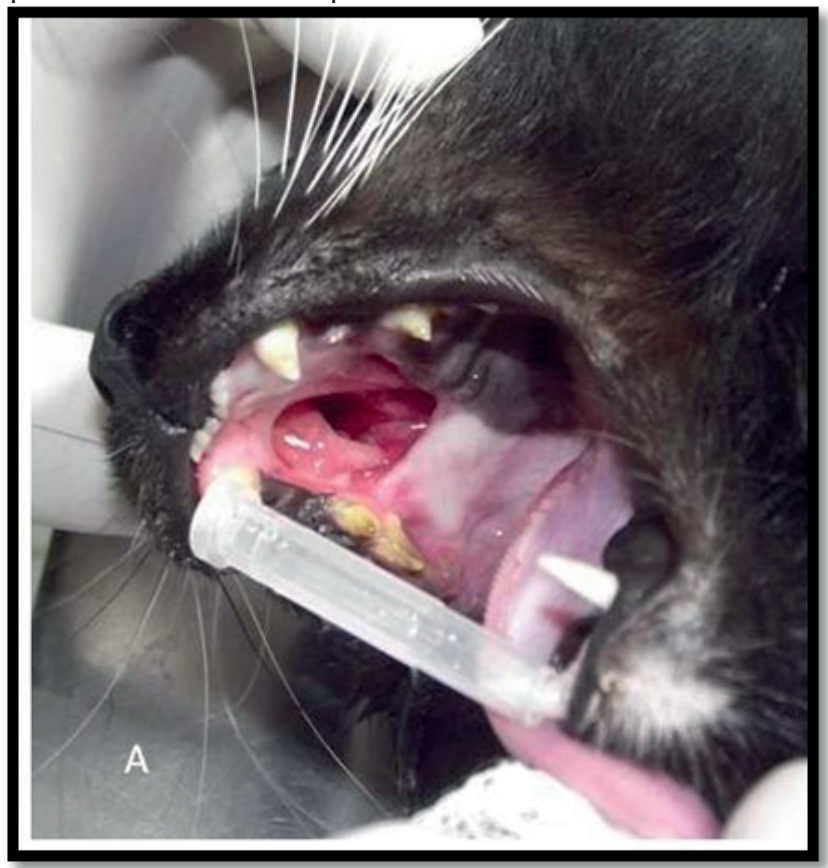

Fonte: arquivo pessoal

Figura 2: Pós- cirúrgico imediato com implante de resina autopolimerizável no defeito palatino. Sonda uretral $n^{\circ} 14$ na cavidade nasal, garantindo a manutenção da via aérea até a polimerização total (seta)

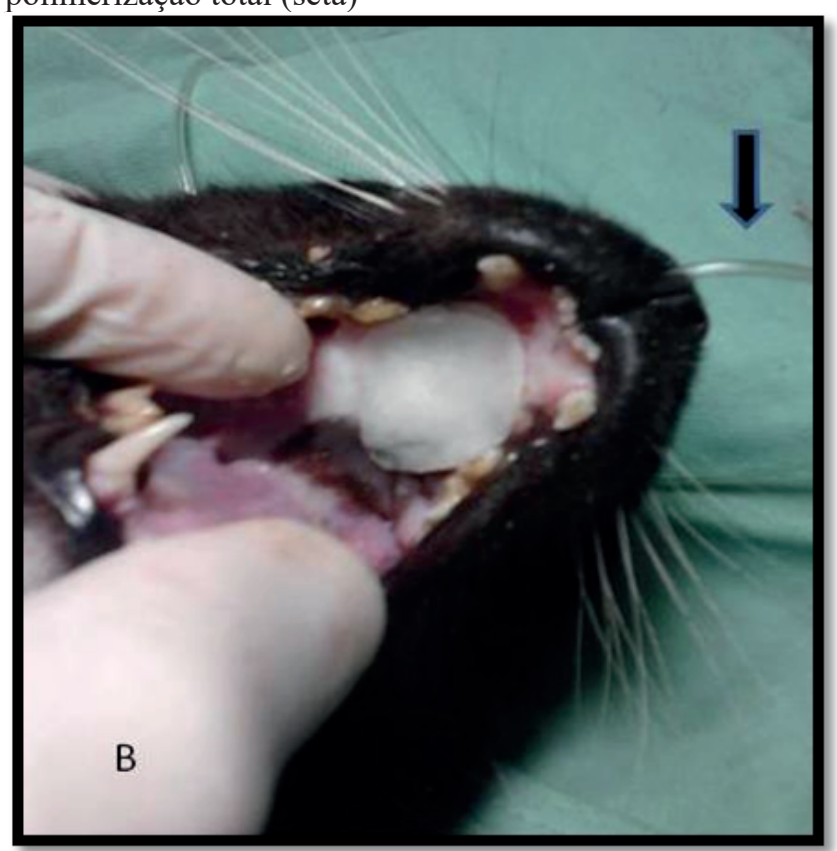

Fonte: arquivo pessoal.
Figura 3: Animal seis meses após procedimento cirúrgico, sem sinais de mobilidade do implante ou inflamação local

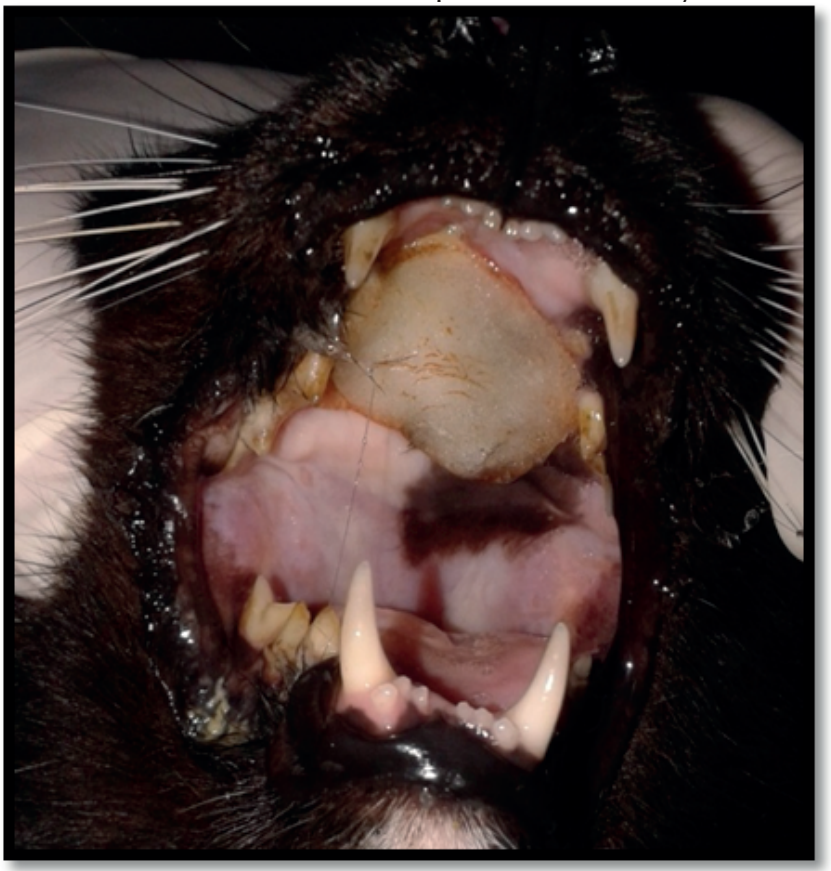

Fonte: arquivo pessoal.

\section{Discussão}

As fendas do palato secundárias normalmente não são diagnosticadas até o aparecimento dos sintomas (FOSSUM, 2002), o que provavelmente ocorreu no caso relatado, já que esta pode ser consequência do trauma sofrido dois anos antes, no entanto é importante destacar a sua possível associação com infecções crônicas, extrações dentárias, intervenções cirúrgicas anteriores, radioterapia ou neoplasias (SIVACOLUNDHU, 2007).

A fenda palatina do presente relato permitia a passagem de alimentos e líquidos para a cavidade nasal, levando aos sinais clínicos comuns dessa afecção tais como rinite, aspiração de alimento, descarga nasal serosa ou muco purulenta, tosse, espirros e halitose (GIOSO,2003; LEE et al., 2006)

O reparo cirúrgico é extremamente necessário diante da extensão do diâmetro da fistula, já que raramente ocorre a cicatrização espontânea da fístula oronasal (BOLSON; PACHALY, 2004).

Houve indicação de avaliação radiográfica torácica pré-cirúrgica devido ao risco de pneumonia por aspiração decorrente da comunicação oronasal (BOLSON; PACHALY, 2004; SILVA, 2006).

Antes do procedimento cirúrgico foi realizada a anti-sepsia com clorexidine a $0,12 \%$ seguindo as recomendações de Goelzer et al. (2003) e Silva et al. (2006), que citam a importância de limpar as vias nasais é indispensável para evitar a contaminação pós-operatória e rinite.

A oclusão da fenda palatina traumática com o uso de resina acrílica autopolimerizável é de fácil execução, aquisição, baixo custo e não invasivo (GIOSO et al., 2001) e foi relatada como uma eficiente técnica já que esta permitiu evolução satisfatória do caso sem causar danosiatrogênicos (COOK et al., 2001). De acordo com Goelzer et al. (2003) o material acrílico é bem tolerado pelos tecidos adjacentes e se mostra eficaz na correção de fístulas oronasais em cães. 
A deiscência da sutura é uma das principais complicação do pós-operatório dos animais que realizam cirurgias de correção da fenda palatina (SOUZA et al., 2007), o que normalmente acontece por conta da tensão na sutura (GRIFFITHS; SULLIVAN, 2001), ou da irritação e inflamação causadas pelo movimento da língua sobre a ferida cirúrgica (LEE et al., 2006), o que não foi visto no presente relato, já que a a prótese palatal colocada não necessitou de suturas para ser fixada, evitando assim quadros de inflamação ou deiscência.

Roehsig et al. (2001) cita a necessidade de elevar-se parcialmente o mucoperiosteo da cavidade oral em toda a circunferência das bordas com o intuito de inserir um fina lamina desse polímero sobre o osso subjacente. No entanto, no presente relato foi feito somente a obstrução com a resina, o que também mostrou ser estável.

As sondas uretrais foram passadas por meio de cada narina para a nasofaringe durante o procedimento, como indicado por Roehsig et al. (2001), impedindo a oclusão total da cavidade nasal enquanto era feito o amoldamento da resina na fenda palatina,permitindo assim a manutenção da cavidade nasal.

A resina acrílica foi moldada diretamente sobre a fistula semelhante ao procedimento utilizado por Roehsiget et al. (2001), já Silva et al. (2009) cita o uso da resina acrílica pré-moldadas, o que evita complicações citadas por Gioso et al. (2001) e Goelzer et al. (2003) como necrose do tecido devido reação exotérmica e posteriormente, pela adesão de placas bacterianas. A utilização da solução salina resfriada e o amoldamento regular da resina, no presente caso, contornou esse problema, visto que não houve surgimento de lesões secundárias à aplicação da resina. Gioso et al. (2001) também recomenda ouso de vaselina ou cera.

No pós-operatório foi realizado a antibioticoterapia para o êxito da reparação da fenda palatina (CONTESINI et al., 2004). O pós-cirúrgico não exigiu restrições alimentares (ROEHSIG et al., 2001) no entanto, optou-se pelo uso da dieta úmida como recomendado por SILVA et al. (2006), com o intuito de estimular o apetite do animal e mesmo facilitar a sua adaptação na mastigação.

A correção cirúrgica realizada tornou o prognóstico favorável, pois o risco de aspiração de alimentos para a via respiratória foi eliminado, com a consequente melhora na ingestão e ganho de peso (SILVA et al., 2006).

\section{Conclusão}

A técnica de oclusão da fenda palatina somente com resina acrílica autopolimerizável promoveu estabilidade adequada e mostrou-se eficaz e de fácil execução para correção de grandes fendas traumáticas em gatos. Portanto, esta se mostra uma boa técnica a ser utilizada em substituição a outros métodos convencionais, principalmente em casos em que suturas ou flapes não são praticáveis.

Diante da pouca literatura relatando a técnica, é necessário um estudo experimental mais aprofundado a fim de se determinar a estabilidade do implante e sua inocuidade.

\section{Referências}

BELOTI, M. M.; OLIVEIRA, P. T.; ROSA, A. L. Engenharia de tecido ósseo em odontologia. Revista ABO Nacional, v. 19, n. 1, p. 164-168, 2011.

BOLSON, J.; PACHALY, J. R. Fístula oronasal em cães (Canis familiaris Linnaeus, 1758) Revisão da literatura. Arquivos de Ciências Veterinárias e Zoologia da UNIPAR. v. 7, n. 1, p. 53-56, 2004.

CONTESINI, E. A. et al. Aspectos clínicos e macroscópicos da palatoplastia imediata com implante de cartilagem da pina auricular, conservada em glicerina a 98\%, após indução experimental de fenda palatina em cães. Ciência Rural, v. 33, n. 1, p. 103-108, 2003.

CONTESINI, E. A. et al. Cartilagem homóloga conservada em glicerina para restauração de fenda de palato duro experimental em cães. Revista da FZVA, v. 11, n. 1, p. 128139, 2004.

COOK, W. T. et al. Influence of an interdental full pin on stability of an acrylic external fixator for rostral mandibular fractures in dogs. American Journal of Veterinary

Research, v. 2, n. 1, p. 10-14, 2001.

FOSSUM, T. W. Cirurgia de Pequenos Animais. São Paulo: Roca, 2002, 177p.

GIOSO, M. A. Exodontia. In: GIOSO, M. A. Odontologia para o clínico de pequenos animais. 5.ed. São Paulo: I editora, 2003, p. 65-72.

GIOSO, M. A. et al. Análise clínica e histológica da utilização da resina acrílica autopolimerizável nas fraturas de mandíbula e maxila e separação da sínfise mentoniana em cães e gatos. Ciência Rural, v. 31, n. 2, p. 291-298, 2001.

GOELZER, L. P. et al. Acrílico auto-polimerizável associado ou não a retalho mucoperiostal simples no tratamento de fístula oronasal experimental em cães. Arquivo Brasileiro de Medicina Veterinária e Zootecnia, v. 55, n. 5 , p. $550-556,2003$.

GRIFFITHS, L.G.; SULLIVAN, M. Bilateral overlapping mucosal single-pedicle flaps for correction of soft palate defects. Journal of the American Animal Hospital Association, v. 37, p. 183-186, 2001.

KUIPERS, R.G. et al. Rapid prototype modeling and customized titanium plate fabrication for correction of a persistent hard palate defect in a dog. Journal of the American Veterinary Medical Association. v. 240, n. 11, p. 1316-1322, 2012.

LEE, J. et al. Application of a temporary palatal prosthesis in a puppy suffering from cleft palate. Journal of Veterinary Science, v. 7, n. 1, p. 93-95, 2006. 
MANFRA MARRETTA, S. Repair of acquired palatal defects. In: VERSTRAETE, F. J.; LOMMER, M. J.

(Editors). Oral and maxillofacial surgery in dogs and cats. Philadelphia: Saunders Elsevier, 2012, p. 363-372.

ROCHA, L.; BECKMAN, B. Soft palate advancement flap for palatal oronasal fistulae. Journal of Veterinary Dentistry, v. 27, p. 132-133, 2010.

ROEHSIG, C. et al. Redução de fenda palatina com resina acrílica autopolimerizável em um gato. A Hora Veterinária, v. 21, n. 121, p. 50-52, 2001.

SILVA, L. M. R. et al. Redução de fenda palatina secundária em um gato. Ciência Veterinária nos Trópicos, v. 9, n. 2/3, p. 97-101, 2006.

SILVA, L. M. R. et al. Redução de fenda palatina, secundária a tumor venéreo transmissível, com obturador palatino. Revista Portuguesa Ciências Veterinárias, v.104, n. 569, p. 77-82, 2009.

SIVACOLUNDHU, R. Use of local and axial pattern flaps for reconstruction of the hard and soft palate. Clinical Techniques in Small Animal Practice, v. 22, p. 61-69, 2007.

SMITH, M. Oronasal fistula repair. Clinical Techniques in Small Animal Practice, v. 15, n. 4, p. 243-250, 2000.

SOUZA, H. J. M. et al.Oclusão de fístula oronasal crónica utilizando a "U"-Plastia da mucosa palatal em gato. Acta Scientiae Veterinariae, v. 35, p. 474-475, 2007.

VERSTRAETE, F. J. M.; LOMMER, M. J.Oral and maxillofacial surgery in dogs and cats. Saunders Elsevier, p.114-129, 2012. 